\title{
Spin-polarized electric currents in quantum transport through tubular two-dimensional electron gases
}

\author{
O. Entin-Wohlman, ${ }^{1,2, \text {, }}$ A. Aharony, ${ }^{1, \text { * }}$ Y. Tokura ${ }^{3}$ and Y. Avishai ${ }^{1}$ \\ ${ }^{1}$ Department of Physics and the Ilse Katz Center for Meso- and Nano-Scale Science and Technology, \\ Ben Gurion University, Beer Sheva 84105, Israel \\ ${ }^{2}$ Albert Einstein Minerva Center for Theoretical Physics, \\ Weizmann Institute of Science, Rehovot 76100, Israel \\ ${ }^{3}$ NTT Basic Research Laboratories, NTT Corporation, Atsugi-shi, Kanagawa 243-0198, Japan
}

(Dated: November 1, 2018)

\begin{abstract}
Scattering theory is employed to derive a Landauer-type formula for the spin and the charge currents, through a finite region where spin-orbit interactions are effective. It is shown that the transmission matrix yields the spatial direction and the magnitude of the spin polarization. This formula is used to study the currents through a tubular two-dimensional electron gas. In this cylindrical geometry, which may be realized in experiment, the transverse conduction channels are not mixed (provided that the spin-orbit coupling is uniform). It is then found that for modest boundary scattering, each step in the quantized conductance is split into two, and the new steps have a non-zero spin conductance, with the spin polarization perpendicular to the direction of the current.
\end{abstract}

PACS numbers: 72.25.-b,72.20.Dp,72.25.Mk

\section{INTRODUCTION}

The conductance of a confined system connected to electronic reservoirs (held at slightly different chemical potentials) can be expressed by its scattering properties, notably the transmission, leading to the celebrated Landauer formula $\underline{1}^{-3}$ When the motion of the electrons is ballistic and the transmission is perfect, the two-terminal conductance is given by

$$
\mathcal{G}=\frac{2 e^{2}}{h} N_{\perp}
$$

where $N_{\perp}$ is the number of conducting channels not including spin. As a function of the energy of the injected electrons, additional channels open successively and the conductance follows a staircase structure, as was indeed observed in experiment. $\underline{\underline{4}}$ When the motion is not ballistic, Eq. (11) is modified, with the number of channels being replaced by the sum over the transmissions among channels,$\frac{3,5}{5}$

Here we extend this picture to include spin-orbit interactions, and derive both charge and spin conductances in terms of the scattering matrix of the mesoscopic system. Our approach is based on scattering theory in which the entire system is represented by its scattering matrix.$\underline{5.6}$ We avoid the ambiguities associated with the definition of spin currents in systems with spin-orbit interactions ${ }^{7}$ by considering scattering through a finite region where these interactions are effective, and computing the currents far away from that region, where these interactions are absent $\underline{\underline{\varepsilon}}$ In this respect our approach differs from those presented in Refs. 9 and 10, which have deduced the conductance from the energy spectrum, or of Ref.11, which employed semiclassical arguments to study spin polarized currents in smooth barriers.

When the electrons are restricted to move in a plane, an asymmetry in the confining potential leads to the appearance of the Rashba spin-orbit interaction. $\underline{12}$ Another source for the spin-orbit coupling is the Dresselhaus mechanism,$\frac{13}{2}$ in particular the cubic one, which might be quite substantial in GaAs nano-structures $\stackrel{14.15}{=}$ The Rashba spin-orbit interaction is in particular interesting, since its strength can be controlled in experiment, by applying gate voltages $\underline{\underline{16}-18}$ From the strictly theoretical point of view, however, there is not much difference between the linear Dresselhaus interaction and the Rashba term, as they are connected by a unitary transformation $\underline{\underline{19}}$ The scattering formalism presented in Sec. $\amalg$ is independent of the type of the spin-orbit coupling.

Spin-orbit interactions, which couple the momentum of the electron to its spin, have attracted much interest due to the possibility to manipulate the spin by electric fields. When electrons are injected from spin-polarized electrodes into a spin-orbit coupled region, they might lose their polarization; this decoherence effect has been studied in Ref. 20. The role of lateral interfaces formed between regions with different spin-orbit couplings 21 in polarizing the spins, and its analogy with optics, has been established in Refs. 22 and 23. However, as direct measurements of the electron spin polarization or of the electron spin accumulation are not easily accomplished (see however Ref. 24), it may be helpful to study the effect of that polarization on the (more easily accessed) electronic charge conductance. At the same time, it is desirable to analyze the spin currents which arise upon the application of a small source-drain bias and explore their possible effect on the charge transport. Previous studies of the conductance through a region where spin-orbit interactions are active had relied on numerical approximations, $\frac{25-28}{-2}$ and consequently some of the conclusions drawn were dictated by a specific choice of 
parameters.

Here we study coherent transport through a mesoscopic hollow cylinder; this geometry enables an exact calculation of the scattering matrix, and reveals its symmetries. In particular we are able to analyze the effect of these symmetries on the spin polarization of the transport current. We present in Sec. II our scattering formalism for spin-dependent transport which follows closely the derivations of the Landauer formula for spinindependent potentials as formulated in Refs. 5 and 6 . We identify there the specific combinations of the elements of the scattering matrix that convey the information on the spin-polarization direction and its magnitude. By carrying out this analysis we are able to show that the transmission matrix of the finite system gives directly the direction and the amount of the spin polarization. Moreover, under not too stringent conditions the conductance itself carries information on the spin polarization. In Sec. III we study the implications of this generalized Landauer formula for a specific system: a coherent mesoscopic hollow cylinder. This system, whose experimental realization is found in the carbon nanotubes, $\stackrel{29}{, 2}$ has recently attracted much interest since the remarkable observation of the effect of the electron motion on the direction of its spin 30 Another experimental realization of this geometry may be found in the core-shell nanowires. 31 Finally, we summarize our conclusions in Sec. IV.

\section{LANDAUER FORMULA FOR SPIN-DEPENDENT TRANSPORT}

In scattering theory ${ }^{5,6}$ the electron field operator (a spinor) is expressed in terms of the scattering states of energy $E$,

$$
\Psi(\mathbf{r}, t)=\int \frac{d E}{2 \pi} e^{-i E t} \sum_{a n \eta} c_{a n \eta}(E) \chi_{a n \eta}(\mathbf{r} ; E) .
$$

(Here and below $\hbar=1$.) In Eq. (2), $\chi_{a n \eta}(\mathbf{r} ; E)$ is a solution of the Schrödinger equation

$$
(E-\mathcal{H}) \chi_{a n \eta}(\mathbf{r} ; E)=0,
$$

with $\mathcal{H}$ being the Hamiltonian of the entire system. It is the scattering state excited by an electron of spin polarization $\eta$ incoming from the $n$th channel of terminal $a$. The operator $c_{a n \eta}(E)\left[c_{a n \eta}^{\dagger}(E)\right]$ destroys (creates) an electron in such a state. The thermal average of these operators (denoted by a bar) is defined by the temperature $T$ and the chemical potential of the reservoirs. For reservoirs of un-polarized electrons,

$$
\overline{c_{a n \eta}^{\dagger}(E) c_{a^{\prime} n^{\prime} \eta^{\prime}}\left(E^{\prime}\right)}=2 \pi \delta\left(E-E^{\prime}\right) \delta_{a a^{\prime}} \delta_{n n^{\prime}} \delta_{\eta \eta^{\prime}} f_{a}(E)
$$

where

$$
f_{a}(E)=\left(e^{\left(E-\mu_{a}\right) / k_{B} T}+1\right)^{-1}
$$

is the Fermi distribution with the chemical potential $\mu_{a}$ of reservoir $a \stackrel{32}{=}$ Equations (2) and (4) are used to obtain the thermal averages of the charge and the spin currents. This is accomplished by writing down the currents in terms of the field $\Psi$, Eq. (2), and then performing the thermal average over the creation and destruction operators, $c$ and $c^{\dagger}$, according to Eq. (4) , 5,6

To avoid cumbersome notations, we confine ourselves to the case of two terminals, located for concreteness along the $x$ direction. Then, all currents flow along $x$ and in the absence of a magnetic field are given by

$$
\begin{aligned}
& I_{x}^{j}(\mathbf{r})=\int \frac{d E}{2 \pi} \sum_{a n \eta} f_{a}(E) \\
& \times \frac{1}{2 m}\left(\left\langle\chi_{a n \eta}(\mathbf{r} ; E)\left|\sigma_{j}\right|\left(-i \frac{\partial}{\partial x}\right) \chi_{a n \eta}(\mathbf{r} ; E)\right\rangle+\mathrm{cc}\right),
\end{aligned}
$$

where $\sigma_{j}$ is the $j$ th Pauli matrix. The charge current, $I_{x}$, is given by Eq. (6) upon identifying $\sigma_{j=0}$ as the unit matrix (and multiplying by the electronic charge $e$ ). For a two-terminal system, the lead index is $a=L$ or $R$, for the left and for the right reservoirs, respectively.

The currents given by Eq. (6) are computed far away from the scattering region, where they are uniform, e.g., on the left side. To this end we represent the scattering states by the incoming and outgoing exciting waves, e.g.,

$$
\begin{aligned}
& \chi_{a n \eta}(x \text { in } L, y, z ; E)=\delta_{a, L} \omega_{a n \eta}^{\text {in }}(\mathbf{r} ; E) \\
& +\sum_{n^{\prime} \eta^{\prime}} \omega_{L n^{\prime} \eta^{\prime}}^{\text {out }}(\mathbf{r} ; E) \mathcal{S}_{L n^{\prime} \eta^{\prime}, a n \eta}(E) .
\end{aligned}
$$

Here, $\omega_{a n \eta}^{\text {in,out }}$ are the exciting waves, incoming or outgoing in channel $n$ of lead $a$ and having the spin polarization $\eta$, normalized to carry a unit flux, and $\mathcal{S}$ is the scattering matrix of our system. The meaning of Eq. (17) is quite transparent: the scattering state which has been excited by a wave incoming in lead $a$ consists, when considered in the left lead $L$, of all waves that are scattered into that lead from lead $a$ (including those that are reflected), as represented by the second term in Eq. (7), and the incoming wave in $a$, in case $a$ coincides with $L . \underline{6}$

Next, the expansion (77) is inserted into Eq. (6), and the integration over the cross section is performed. As a result, $I_{x}^{j}$ becomes spatially-independent,

$$
\begin{aligned}
& I_{x}^{j}=\int \frac{d E}{2 \pi} \sum_{a} f_{a}(E) \operatorname{Tr}\left\{\delta_{a, L} \sigma_{j}-\sum_{n n^{\prime}} \mathcal{M}_{L n^{\prime}, a n}(E) \sigma_{j}\right\} \\
& =\int \frac{d E}{2 \pi}\left(f_{L}(E)-f_{R}(E)\right) \operatorname{Tr} \sum_{n n^{\prime}} \mathcal{M}_{L n^{\prime}, R n}(E) \sigma_{j},
\end{aligned}
$$

where the trace is carried out in spin space. Here we have introduced the definition

$$
\mathcal{M}_{L n^{\prime}, a n}(E) \equiv \mathcal{S}_{L n^{\prime}, a n}(E) \mathcal{S}_{L n^{\prime}, a n}^{\dagger}(E)
$$

where $\mathcal{S}_{L n^{\prime}, R n}$ denotes an entry of the scattering matrix which is a $2 \times 2$ matrix in the spin space. The second equality in Eq. (8) is derived from the unitarity of the 
scattering matrix. Note that $I_{x}^{j}$ vanishes when there is no bias voltage, $\mu_{L}=\mu_{R}$. Although it appears as if pertains for general values of the bias voltage, Eq. (8) is strictly valid only in the linear-response regime, in where $\mu_{L}-\mu_{R}$ approaches zero. A detailed account of the subtleties associated with the Landauer formula can be found in Ref. 2.

The general expression Eq. (8) for the charge and the spin currents is our central result in this section. In the small-bias limit it yields the linear charge and spin conductances. To express those, it is useful to note that the transmission from channel $n$ to channel $n^{\prime}$ is determined by the matrix $\mathcal{M}$, Eq. (91). Since this matrix is obviously hermitian, it can be decomposed into a scalar and a vector,$\underline{20}$

$$
\mathcal{M}_{L n^{\prime}, R n}(E)=\frac{1}{2}\left(\mathcal{T}_{n^{\prime} n}(E)+\mathbf{V}_{n^{\prime} n}(E) \cdot \boldsymbol{\sigma}\right),
$$

where both $\mathcal{T}_{n^{\prime} n}$ and $\mathbf{V}_{n^{\prime} n}$ are real. The two real eigenvalues of this matrix are

$$
\lambda_{n^{\prime} n}^{ \pm}(E)=\frac{1}{2}\left(\mathcal{T}_{n^{\prime} n}(E) \pm\left|\mathbf{V}_{n^{\prime} n}(E)\right|\right) .
$$

Their corresponding eigenvectors are spinors fully polarized along $\pm \mathbf{V}_{n^{\prime} n}$. The total transmission between these two channels is given by

$$
\mathcal{T}_{n^{\prime} n}=\operatorname{Tr}\left[\mathcal{M}_{L n^{\prime}, R n}\right]=\lambda_{n^{\prime} n}^{+}+\lambda_{n^{\prime} n}^{-},
$$

while the spin transmission is

$$
\operatorname{Tr}\left[\mathcal{M}_{L n^{\prime}, R n} \boldsymbol{\sigma}\right]=\mathbf{V}_{n^{\prime} n},
$$

with

$$
\left|\mathbf{V}_{n^{\prime} n}\right|=\lambda_{n^{\prime} n}^{+}-\lambda_{n^{\prime} n}^{-} .
$$

As is well known, (see e.g., Refs. 11 and 22) spin-orbit interactions may turn one of the modes (for each energy) to be evanescent; when this happens, the eigenvalue belonging to that mode, $\lambda^{-}$, is vanishingly small. As a result, the (perfect) conductance is reduced to $\lambda^{+} \simeq 1$ (in units of $e^{2} / h$ ), while the current becomes almost fully spin-polarized, its respective conductance being $\left|\mathbf{V}_{n n^{\prime}}\right| \simeq \lambda_{n n^{\prime}}^{+} \simeq 1$. On the other hand, when none of the modes is evanescent, one has $\lambda_{n n^{\prime}}^{+} \simeq \lambda_{n n^{\prime}}^{-} \simeq 1$, leading to $\mathcal{G}=2 e^{2} / h$ and zero spin polarization for the perfect conductor.

\section{CHARGE AND SPIN TRANSPORT THROUGH A COHERENT TUBULAR TWO-DIMENSIONAL ELECTRON GAS}

In order to illustrate the general features of the scattering matrix as embodied in Eqs. (10)-(14), we study a specific example: transport through a two-dimensional stripe, of width $2 d$ (along $x$ ), in which the electrons are subject to the Rashba spin-orbit interaction. The interfaces at $x= \pm d$ are parallel to the $y$-axis; effects of interfacial scattering are included by a repulsive deltafunction potential located at the interface. Elsewhere, the electrons move ballistically. Such a potential is characterized by a single parameter, $\zeta$ (measured in momentum units). When $\zeta$ is very large, the interface approaches the tunnel-junction limit. This model becomes particularly transparent when a periodic boundary condition along the $y$ axis is assumed; then the spin-orbit interaction does not mix the transversal modes. This model system, whose realization may be found in nanotubes (see in particular Ref. 30) is depicted in Fig. 11. For cylinders, the coordinate $y$ represents the azimuthal angle around the cylinder (with perimeter $\mathcal{L}$ ), and the coordinate $z$ is radial, i.e. perpendicular to the surface of the cylinder.

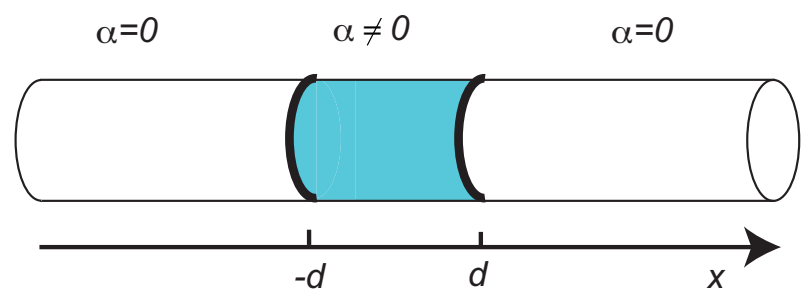

FIG. 1: (Color online) A schematic representation of our model: a hollow cylinder, containing a region, $|x| \leq d$, where a spin-orbit interaction, of strength $\alpha$, is active. The electrons are moving on the surface of the tube ballistically, and thus mode mixing is hindered. Short-range boundary scattering, of strength $\zeta$, separate the spin-orbit coupled region from the free regions. The boundaries in-between these regions are marked by thick solid lines.

The scattering matrix is computed by solving for the scattering states of the Hamiltonian

$$
\begin{aligned}
& \mathcal{H}=\frac{\mathbf{p}^{2}}{2 m}+\frac{\zeta}{m}(\delta(x+d)+\delta(x-d)) \\
& +\frac{\alpha}{2 m}(\Theta(d-x) \Theta(x+d) \mathbf{p} \cdot(\hat{z} \times \boldsymbol{\sigma})+\mathrm{hc}),
\end{aligned}
$$

where $\mathbf{p}$ is the (two-dimensional) momentum operator and $\alpha$ is the spin-orbit coupling (in momentum units). With the periodic boundary condition, of period $\mathcal{L}$, the wave functions vary along $y$ as $\exp [i q y]$, and the transverse momentum is quantized,

$$
q= \pm 2 \pi n / \mathcal{L}, \quad n=0,1,2, \ldots .
$$

Below, we measure all momenta (including $\alpha$ and $\zeta$ ) in units of $\hbar / \mathcal{L}$. The $x$ component of the wave vector is

$$
k=\sqrt{2 m E-q^{2}}, \quad|x| \geq d,
$$

for both spin components in the absence of the spin-orbit interaction, and

$$
\begin{gathered}
k_{u}=\sqrt{\left(p_{S} \pm \alpha\right)^{2}-q^{2}} \\
l
\end{gathered}
$$


for waves in the range $|x|<d$ where the spin-orbit interaction is effective 11 Here,

$$
p_{S}=\sqrt{2 m E+\alpha^{2}} .
$$

As $q^{2} \leq 2 m E$, the wave vector $k_{u}$ is always real; in contrast, $k_{l}$ is purely imaginary for $|q|>p_{S}-\alpha$ (we assume $\alpha>0$ ), and then one of the waves in the region $|x|<d$ is evanescent 11,22

Following the standard procedure of matching boundary conditions at the two interfaces $x= \pm d$ (allowing for the delta-function potentials) $\underline{21}, 33$ the scattering matrix of each channel takes the form

$$
\mathcal{S}=-1+\left[\begin{array}{cc}
\mathcal{S}_{+}+\mathcal{S}_{-} & \left(\mathcal{S}_{+}-\mathcal{S}_{-}\right) \sigma_{x} \\
\sigma_{x}\left(\mathcal{S}_{+}-\mathcal{S}_{-}\right) & \sigma_{x}\left(\mathcal{S}_{+}+\mathcal{S}_{-}\right) \sigma_{x}
\end{array}\right]
$$

where

$$
\mathcal{S}_{ \pm}^{-1}=\frac{1}{k}\left(k+2 i \zeta+i X_{ \pm}-i \sigma_{z}\left(q-Z_{ \pm}\right)+i \sigma_{x} Y_{ \pm}\right) .
$$

The functions $X_{ \pm}, Y_{ \pm}$, and $Z_{ \pm}$are all real, independent of whether $k_{l}$ [see Eq. (18)] is real or imaginary,

$$
\begin{aligned}
& X_{ \pm}=\frac{p_{S}}{D_{0}}\left(\frac{S_{l}\left[C_{u}\left(p_{S}-\alpha\right) \pm q\right]}{k_{l}}+\frac{S_{u}\left[C_{l}\left(p_{S}+\alpha\right) \mp q\right]}{k_{u}}\right), \\
& Y_{ \pm}=\frac{p_{S}}{D_{0}}\left(\frac{S_{u}\left[q C_{l} \mp\left(p_{S}+\alpha\right)\right]}{k_{u}}-\frac{S_{l}\left[q C_{u} \pm\left(p_{S}-\alpha\right)\right]}{k_{l}}\right) \\
& Z_{ \pm}=\frac{p_{S}}{D_{0}}\left(2 q p_{S} \frac{S_{l} S_{u}}{k_{l} k_{u}} \pm\left(C_{l}-C_{u}\right)\right),
\end{aligned}
$$

where

$$
D_{0}=1-C_{l} C_{u}+\frac{p_{S}^{2}-\alpha^{2}+q^{2}}{k_{l} k_{u}} S_{l} S_{u} .
$$

Here we use the shorthand notations

$$
S_{l, u} \equiv \sin \left(2 k_{l, u} d\right), \quad C_{l, u} \equiv \cos \left(2 k_{l, u} d\right) .
$$

When the transversal channels are not mixed, then the total charge and spin transmissions are given by

$$
\mathcal{T}=\sum_{n} \mathcal{T}_{n n}, \text { and } \quad \mathbf{V}=\sum_{n} \mathbf{V}_{n n}
$$

see Eqs. (8), (12), and (13). The scattering matrix Eq. (20) is unitary,$\frac{34}{4}$ and is self-dual ${ }^{35}$ upon changing $q$ to $-q$. (Other symmetries of the scattering matrix, in particular in the presence of the Zeeman interaction, are discussed in Refs. 36 and 37.) The self-duailty symmetry of the scattering matrix implies that upon summing over all channels, as indicated by Eqs. (25) [i.e., over positive and negative values of the transverse wave vector $q$, see Eq. (16)] the contributions of the $x$ and $z$ directions of the polarization are cancelled, and the net spin transmission $\mathbf{V}$ is along the $y$ direction, normal to the direction of the current $\stackrel{11.28}{\underline{1}}$
Figures 2,4 were drawn using Eq. (20). The curves in Fig. 2 are computed for entirely transparent boundaries $(\zeta=0)$; indeed, the transmission in the absence of the spin-orbit coupling has the familiar perfect staircase structure, Eq. (11). Due to the choice of periodic boundary conditions in the transverse direction, the perfect transmission without the spin-orbit interaction becomes $2 N_{\perp}=2(2 n+1)$, where $n=0,1,2, \ldots$ is the channel number. The transmission computed in the presence of that coupling follows roughly this pattern, but with two distinct new features. Firstly, it shows interference oscillations. These arise since the spin-orbit interaction plays the role of a potential step at $|x|<d \stackrel{38}{ }$ One notes the complete vanishing of the spin conductance in the leftmost part of the plot. There, the energy is too low to support a nonzero transverse momentum $q$, and consequently the motion becomes effectively onedimensional. In such a case, one may handle the effect of the spin-orbit interaction by a gauge transformation which multiplies the wave function by $\exp \left[2 i p_{S} d\right]$. Since this phase factor is the sole effect of the spin-orbit interaction, the spin-polarization vanishes completely: $\underline{\underline{39}}$ (Oscillations as a function of the spin-orbit coupling strength have been discussed in Ref. 40.) When $q \neq 0$ and the motion restores its two-dimensional character, this gauge transformation is no longer possible, and indeed there appears spin conductance. The oscillations in the charge conductance, resulting from the potential-step aspect of the spin-orbit interaction, do persist.

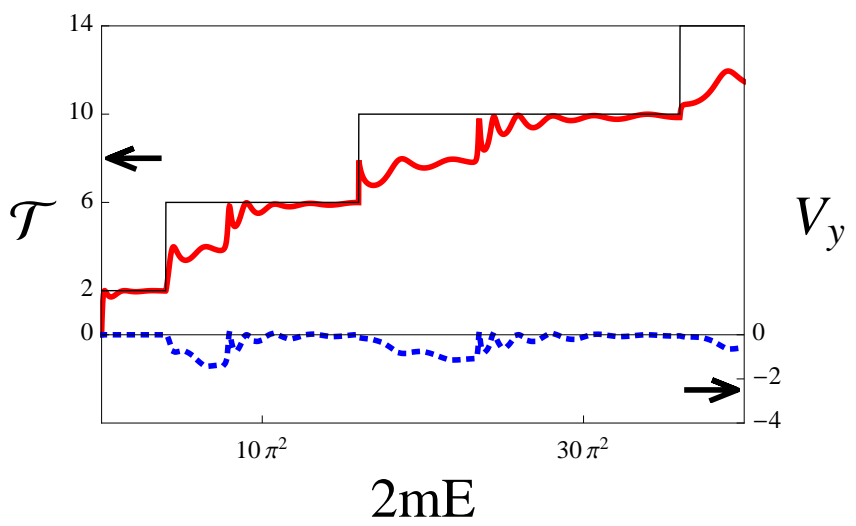

FIG. 2: (Color online) The transmission $\mathcal{T}$ through a stripe with spin orbit interaction (thick line), the $y$ - component of the spin transmission $V_{y}$ (dotted line), and the staircase structure obtained in the absence of the spin-orbit coupling (thin line) in a perfect system, as a function of the energy. Here, the spin-orbit strength is $\alpha=0.9 \pi$.

The second prominent feature that the spin-orbit interaction induces in the conductance pattern originates from the possibility to have evanescent waves. At the beginning of each of the $n \neq 0$ steps [see Eq. [16)], one of the waves pertaining to that energy becomes evanescent, since using Eq. (18) one has

$$
k_{l}=i \kappa
$$


where $\kappa$ is real. Then, $C_{l}$ and $-i S_{l}$ of Eqs. (24) are both of order $0.5 \exp [2 \kappa d]$, making the determinant of the transmission matrix $\mathcal{M}$, Eqs. (10) and (20), exponentially small. This implies in turn that one of the eigenvalues, Eqs. (11), i.e. $\lambda_{n n}^{-}$, is very small. As a result, it follows from Eq. (12) that the (perfect) transmission is reduced to $\mathcal{T}_{n n} \simeq \lambda_{n n}^{+} \simeq 1$. Concomitantly, the polarization magnitude, $\left|\mathbf{V}_{n n}(q)\right|$, [see Eq. (14)] is also equal to $\lambda_{n n}^{+} \simeq 1$. However, upon adding $\mathbf{V}(q)+\mathbf{V}(-q)$ the resulting vector $\mathbf{V}$ is along the $y$-axis, as shown in Fig. 2. This $y$-component decreases gradually from 0 towards -1 as the energy increases in the steps where $\mathcal{T} \simeq 4 n$. At higher energies, both $k_{u}$ and $k_{l}$ are real, and the (perfect) transmission becomes $\mathcal{T}_{n n}=\lambda_{n n}^{+}+\lambda_{n n}^{-} \approx 2$, while the spin transmission becomes $\left|\mathbf{V}_{n n}(q)\right|=\lambda_{n n}^{+}-\lambda_{n n}^{-} \approx 0$. For the parameters used in Figs. 2 and 3 the contributions of all the steps except the highest one are in this latter regime, and therefore each of them contributes 2 (if $q=0$ ) or 4 (if $q \neq 0$ ) to the transmission and 0 to the spin transmission. Thus, the charge conductance exhibits $2(2 n+1)$ and $4 n$ multiples of $e^{2} / h$, while the spin transmission is roughly between 0 and -1 . For higher values of $\alpha$, one of the waves may remain evanescent until the next step begins, yielding only $4 n$ steps in the transmission 11 In our dimensionless units, this condition becomes $\alpha>(2 n+1) \pi / n$.

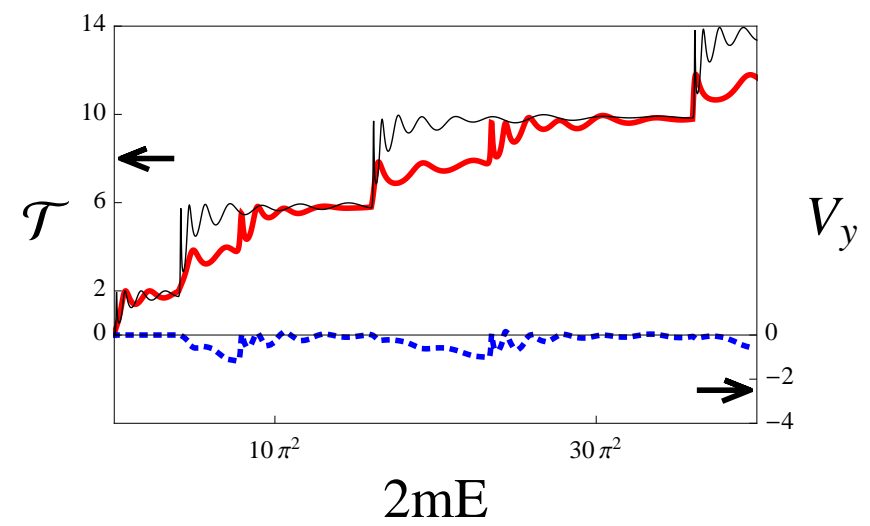

FIG. 3: (Color online) Same as Fig. 2. but in the presence of potential barriers at the boundaries, $\zeta=1.2$. Here, $\alpha=0.9 \pi$.

Repeating the computation for finite barriers at the two interfaces [see Fig. 3] shows that a modest amount of interface scattering is not detrimental, and the two main features discussed above are still detected. Even more interesting are the curves shown in Fig. 4. Here, the amount of scattering at the interfaces has been increased such that the staircase structure of the charge conductance, both in the presence and in the absence of the spin-orbit coupling, is smeared; since the conduc- tance is not quantized, the spin-polarization is small (but still negative) for all values of the energy.

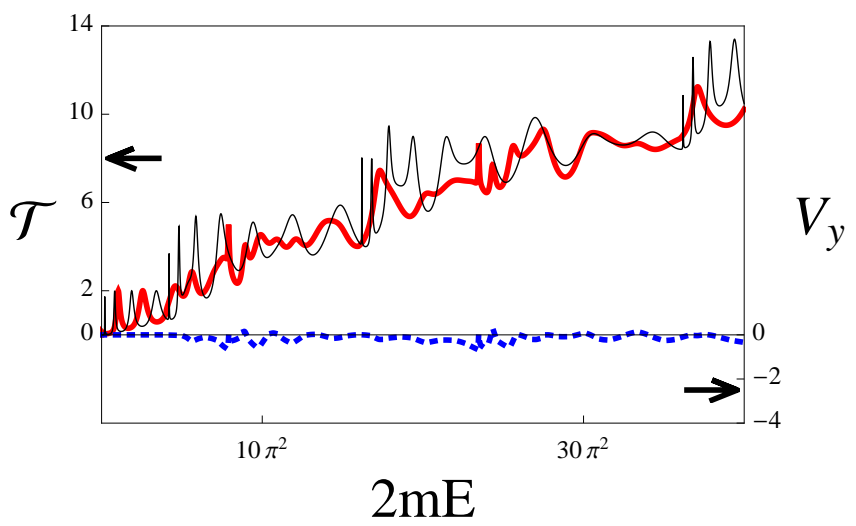

FIG. 4: (Color online) Same as Fig. 2 but with stronger boundary scattering, $\zeta=4$, and $\alpha=.9 \pi$.

\section{CONCLUDING REMARKS}

In conclusion, we have derived a Landauer formula for the charge and the spin conductances. The correlations between the two conductances and their quantization have been demonstrated for transport through cylinders with stripes which have nonzero spin-orbit interactions. Measurements of the charge transmission can thus yield information on the spin polarization. It would be interesting to check these predictions experimentally, in particular on the new set-ups of core-shell nanowires or made of carbon nanotubes.

\section{Acknowledgments}

We thank P. Silvestrov for helpful comments. AA and OEW acknowledge support by the binational IsraelUnited States foundation, by a grant from the German Federal Ministry of Eduction and Research (BMBF) within the framework of the German-Israeli Project Cooperation (DIP), and the hospitality of NTT Basic Research Laboratory at Atsugi-shi, Japan. YA acknowledges partial support from the ISF, and the hospitality of the Graduate School of Mathematical Science at the University of Tokyo. 
21, 863 (1970).

2 Y. Imry and R. Landauer, Rev. Mod. Phys. 71, S306 (1999).

3 Y. Imry, Introduction to Mesoscopic Physics, 2nd ed. (Oxford University Press, Oxford, 2002).

4 D. A. Wharam, T. J. Thornton, R. Newbury, M. Pepper, H. Ahmed, J. E. F. Frost, D. G. Hasko, D. C. Peacock, D. A. Ritchie, and G. A. C. Jones, J. Phys. C21, L209 (1988); B. J. van Wees, H. van Houten, C. W. J. Beenakker, J. G. Williamsom, L. P. Kouwenhoven, D. van der Marel, and C. T. Foxon, Phys. Rev. Lett. 60, 848 (1988).

${ }^{5}$ M. Büttiker, Phys. Rev. B 46, 12485 (1992).

6 Y. Levinson, Phys. Rev. B 61, 4748 (2000).

7 J. Shi, P. Zhang, D. Xio, and Q. Niu, Phys. Rev. Lett. 96, 076604 (2006).

8 B. Nikolić, L. P. Zârbo, and S. Souma, Phys. Rev. B 73, 075303 (2006).

9 A. V. Moroz and C. H. W. Barnes, Phys. Rev. B 60, 14272 (1999).

10 Y. V. Pershin, J. A. Nesteroff, and V. Privman, Phys. Rev. B 69, 121306(R) (2004).

11 P. G. Silvestrov and E. G. Mischenko, Phys. Rev. B 74, 165301 (2006).

12 E. I. Rashba, Fiz. Tverd. Tela (Leningrad) 2, 1224 (1960) [Sov. Phys. Solid State 2, 1109 (1960)]; Yu. A. Bychkov and E. I. Rashba, Pis'ma Zh. Eksp. Teor. Fiz. 39, 66 (1984) [Sov. Phys. JETP Lett. 39, 78 (1984)].

13 G. Dresselhaus, Phys. Rev. 100, 580 (1955).

14 J. J. Krich and B. I. Halperin, Phys. Rev. Lett. 98, 226802 (2007).

15 A. A. Kovalev, M. F. Borunda, T. Jungwirth, L. W. Molenkamp, and J. Sinova, Phys. Rev. B 76, 125307 (2007).

16 T. Koga, J. Nitta, T. Akazaki, and H. Takayanagi, Phys. Rev. Lett. 89, 046801 (2002).

17 M. König, A. Tschetschekin, E. M. Hankiewicz, J. Sinova, V. Hock, V. Daumer, M. Schäfer, C. R. Becker, H. Buhmann, and L. W. Molenkamp, Phys. Rev. Lett. 96, 076804 (2006).

18 T. Bergsten, T. Kobayashi, Y. Sekine, and J. Nitta, Phys. Rev. Lett. 97, 196803 (2006).

19 E. I. Rashba and V. I. Sheka, in Landau Level Spectroscopy, G. Landwehr and E. I. Rashba, eds. (Elsevier, Amsterdam, 1991).

20 B. Nikolić and S. Souma, Phys. Rev. B 71, 195328 (2005); R. L. Dragomirova and B. K. Nikolić, Phys. Rev. B 75, 085328 (2007).

21 V. A. Sablikov, A. A. Sukhanov, and Y. Ya. Tkach, Phys. Rev. B 78, 153302 (2008).

22 M. Khodas, A. Shekhter, and A. M. Finkel'stein, Phys. Rev. Lett. 92, 086602 (2004); A. Shekhter, M. Khodas, and A. M. Finkel'stein, Phys. Rev. B 71, 125114 (2005).

${ }^{23}$ V. Marigliano Ramaglia, D. Bercioux, V. Cataudella, G. De Filippis, and C. A. Perroni, J. Phys.: Condens. Matter 16, 9143 (2004).

24 I. Adagideli, G. E. W. Bauer, and B. I. Halperin, Phys. Rev. Lett. 97, 256601 (2006).

25 F. Mireles and G. Kirczenow, Phys. Rev. B 64, 024426 (2001).

26 Shi-Liang Zhu, Z. D. Wang, and Lian Hu, J. Appl. Phys. 91, 6545 (2002).

27 M. Governale and U. Zülicke, Solid State Comm. 131, 581 (2004).

28 M. Eto, T. Hayashi, and Y. Kurotani, J. Phys. Soc. Jpn. 74, 1934 (2005).

29 The spin-orbit interaction in nanotubes is not predominantly of the Rashba type used in Sec. III see T. Ando, J. Phys. Soc. Jpn. 69, 1757 (2000). However, the results of Sec. II are valid for these systems.

${ }^{30}$ F. Kuemmeth, S. Ilani, D. C. Ralph, and P. L. McEuen, Nature 452, 448 (2008).

31 L. J. Lauhon, M. S. Gudiksen, D. Wang, and C. M. Lieber, Nature 420, 57 (2002); J. Noborisaka, J. Motohisa, S. Hara, and T. Fukui, Appl. Phys. Lett. 87, 093109 (2005); G. Zhang, K. Tateno, T. Sogawa, and H. Nakano, Appl. Phys. Express 1, 063003 (2008); J. W. W. van Tilburg, R. E. Algra, W. G. G. Immink, M. Verheijen, E. P. A. M. Bakkers, and L. P. Kouwenhoven, Semicond. Sci. Technol. 25, 024011 (2010).

32 The extension of the formalism presented in Sec. II to the case of spin-polarized leads is rather straightforward.

33 When writing down the boundary conditions, one should match the covariant momentum-which includes the spinorbit contribution.

34 Since $\mathcal{S}_{ \pm}^{-1}+\left(\mathcal{S}_{ \pm}^{\dagger}\right)^{-1}=2$, the scattering matrix Eq. (20) is unitary.

35 C. W. J. Beenakker, Rev. Mod. Phys. 69, 731 (1997).

36 F. Zhai and H. Q. Xu, Phys. Rev. Lett. 94, 246601 (2005).

37 P. Brusheim and H. Q. Xu, Phys. Rev. B 74, 233306 (2006).

38 This effect resembles in a way the appearance of Andreev reflections at the interface between a superconductor and a normal metal, see G. E. Blonder, M. Tinkham, and T. M. Klapwijk, Phys. Rev. B 25, 4515 (1982).

39 A. A. Kiselev and K. W. Kim, Phys. Rev. B 71, 153315 (2005)]; note that Sánchez and Serra [Phys. Rev. B 74, 153313 (2006)] do not find polarization for an harmonic confining potential along $y$.

40 A. G. Mal'shukov, V. V. Shlyapin, and K. A. Chao, Phys. Rev. B 66, 081311 (2002); C-H Chang, A. G. Mal'shukov, and K. A. Chao, Phys. Lett. A 326, 436 (2004). 